\title{
Numerical Study of Bird Flu Infection Process Within a Poultry Farm with Age Structured Model
}

\author{
Tertia Delia Nova ${ }^{1}$, Arrival Rince Putri ${ }^{2}$, and Masaji Watanabe ${ }^{3}$ \\ ${ }^{1}$ Faculty of Animal Science, Andalas University, Padang, Indonesia \\ ${ }^{2}$ Faculty of Mathematics and Natural Sciences, Andalas University, Padang, Indonesia \\ ${ }^{3}$ Graduate School of Environmental and Life Science, Okayama University, Okayama, Japan
}

\section{Abstract}

Bird flu infection processes within a poultry farm were studied analytically and numerically. A mathematical model proposed in a previous study was reformulated with consideration of age structure. The age structure model for a susceptible population and an infected population is described. Numerical results show that essential factors of security against bird flu are vaccination and removal of infected bird.

Corresponding Author:

Tertia Delia Nova

watan-m@okayama-u.ac.jp

Received: 19 February 2019

Accepted: 5 March 2019

Published: 16 April 2019

Publishing services provided by

Knowledge E

(c) Tertia Delia Nova et al. This article is distributed under the terms of the Creative Commons Attribution License, which permits unrestricted use and redistribution provided that the original author and source are credited.

Selection and Peer-review under the responsibility of the ICBSA Conference Committee.
Keywords: bird flu, mathematical model, numerical simulation, age structure

\section{Introduction}

The loss of domestic birds due to bird flu has been a serious issue to poultry farmers ever since the disease prevailed worldwide in 2003. The source of the disease is an influenza virus $\mathrm{H} 5 \mathrm{~N} 1$. Forms of infection with $\mathrm{H} 5 \mathrm{~N} 1$ is classified into low pathogenic form and highly pathogenic form. The infection with the highly pathogenic form spreads rapidly over a poultry farm, and causes serious symptoms to domestic birds, which eventually lead to death. Even if infection of only one bird with H5N1 is detected, all the birds in the farm become subject to culling. The losses due to culling of domestic birds have been causing enormous damages to the poultry industry.

A bird flu infection process within a poultry farm involves the source of disease (influenza virus), the host (poultry), and the medium (environment). Once bird flu attacks a poultry farm, some birds die at the early stage of an infection process, and some others may live longer. Regardless of being alive or dead, infected birds remain as sources of infection, unless they are completely removed from the farm. Those factors were incorporated into formulation of mathematical model for populations of susceptible 
of virus concentration to unknowns [3-6]. Those previous studies show that a proper vaccination and a proper removal of infected birds are essential for security of a poultry farm against bird flu.

In this study, age structure of domestic birds was incorporated into formulation of a mathematical model. In an egg production process, entire population of domestic birds is maintained at the appropriate capacity by supply of six-month old birds for vacancies created by removal of thirty-month old birds. Thus entire population is distributed over the age interval from six months to thirty months. In the following sections, mathematical model is described and some analytical results are presented. Numerical results are also presented.

\section{Materials and Methods}

\subsection{Modeling bird flu infection process with age structure}

When bird flu intrudes into a poultry farm, domestic birds are divided into the class of healthy birds susceptible to infection and the class of infected birds. The SI model was proposed in studies of the population of susceptible individuals and the population of infected individuals [7]. The $S /$ model is inappropriate for susceptible and infected populations of poultry farms, where the entire population is regulated. In a production process of a poultry farm, the entire population of domestic birds is kept at the capacity of the farm with supply of new birds for vacancies. Let $X(t)$ and $Y(t)$ denote the population of susceptible birds and the population of infected birds, respectively, at time $t$, and Let $c$ be the capacity of the farm. Then the rate of supply of new birds is $a[c-(X(t)+Y(t))]$, where $a$ is a positive constant. The number of susceptible birds infected per unit time is proportional to the numbers of susceptible birds and infected birds, $\omega X(t) Y(t)$, where $\omega$ is a positive constant. The infected birds do not recover from the disease. Some birds die at the early stage of infection and others stay alive longer. Regardless of being alive or dead, infected birds are virus carriers unless they are removed from the population. The removal rate of infected birds is proportional to their population, and it is expressed by $-m Y(t)$, where $m$ is the removal rate. The following system (1), (2) was proposed [1].

$$
\begin{gathered}
\frac{d X}{d t}=a[c-(X+Y)]-\omega X Y, \\
\frac{d Y}{d t}=\omega X Y-m Y,
\end{gathered}
$$

In this study, age structure was considered in formulation of susceptible birds and infected birds. In an egg production processes, entire population of domestic birds is 
maintained at the capacity of the farm by a supply of six-month old birds for vacancies created by removal of thirty-month old birds. Suppose that domestic birds in a poultry farm is distributed over an age interval $[q, r]$. Here $q[\mathrm{mth}]$ is the age of fresh birds that are supplied for vacancies, and $r$ [mth] is the age of birds that are removed from the egg production process. Denote by $X(a, t)$ the numbers of susceptible birds of age $a$ [mth] at time $t$ [mth], and $Y(a, t)$ the numbers of infected birds of age $a$ [mth] at time $t$ [mth], respectively. The rate of infection of susceptible birds of age $a$ at time $t$ is proportional to the product of $X(a, t)$ and the total number of infected birds

$$
\int_{q}^{r} Y(a, t) d a
$$

and the removal rate of infected birds of age is at time $t$ is proportional to $Y(a, t)$

The following system of equations are proposed.

$$
\begin{gathered}
\frac{\partial X}{\partial t}+\frac{\partial X}{\partial a}=-\omega X \int_{q}^{r} Y(a, t) d a, \\
\frac{\partial Y}{\partial t}+\frac{\partial Y}{\partial a}=\omega X \int_{q}^{r} Y(a, t) d a-m Y,
\end{gathered}
$$

System of equations (3), (4) is associated with the initial conditions,

$$
X(a, 0)=X_{0}(a), \quad Y(a, 0)=Y_{0}(a) .
$$

Vacancies are replaced with supply of susceptible birds of age $q$. So the system of equations (3), (4) is associated with the boundary condition,

$$
X(q, t)=c-\int_{q}^{r}[X(a, t)+Y(a, t)] d a, \quad Y(q, t)=0 .
$$

\subsection{Stationary state population of susceptible birds and infected birds}

Stationary points of system (1), (2) are constant solutions. For fixed but arbitrary positive values of $a, c, \omega$, and $m$, there are two stationary points,

$$
(X, Y)=(c, 0) .
$$

and

$$
(X, Y)=\left(\frac{m}{\omega}, \frac{a(c \omega-m)}{\omega(a+m)}\right) .
$$

The stationary point (7) corresponds to the state of no infection, in which no bird is infected. The stationary point (8) corresponds to an endemic state in which a part 
of population is always infected. The stationary point (8) is practical provided its $y$ component is positive, that is,

$$
c \omega-m>0,
$$

while it is unpractical for $c \omega-m<0$. The stationary points (7) and (8) coincide for $c \omega-m=0$. When the stationary point (7) is asymptotically stable, the state returns to it after disturbance due to intrusion of bird flu. The stationary point (7) is unstable under the condition (9), and it is asymptotically stable for $c \omega-m<0$. The stationary point (8) is asymptotically stable under the condition (9). It is unstable for $c \omega-m<0$ [2].

The initial boundary value problem (3) - (6) has a constant solution. Suppose that $X(a, t)=\xi, Y(a, t)=\eta(q \leq a \leq s, t \geq 0)$ is a constant solution of the system (3) - (6), where $\xi$ and $\eta$ are nonnegative constants.

Equations (3) and (4) lead to

$$
\begin{gathered}
-\omega(r-q) \xi \eta=0, \\
{[\omega(r-q) \xi-m] \eta=0 .}
\end{gathered}
$$

System of equations (10), (11) implies $\eta=0$, and the boundary condition (6) leads to

$$
\xi=c-(r-q) \xi .
$$

The solution of equation (12) is $\xi=c /(1+r-q)$, and

$$
(X, Y)=\left(\frac{c}{1+r-q}, 0\right)
$$

is a constant solution of the initial boundary value problem (3) - (6).

\section{Results}

\subsection{Numerical solution of equations for susceptible population and infected populatio}

The initial boundary value problem (3) - (6) was analyzed numerically for $q=6.0 s=30.0$, $c=1.0$, and $\omega=1.0$. Figs. $1-8$ shows profiles of numerical solutions of the initial boundary value problem (3) - (6). Figs. 1 - 4 shows the profiles of numerical solutions at $t=0, t=10, t=20$, and $t=30[\mathrm{mth}]$ in case $c \omega-m<0$. Figs. $5-8$ shows the profiles of numerical solutions at $t=0, t=10, t=20$, and $t=30[\mathrm{mth}]$ in case $c \omega-m>0$. 


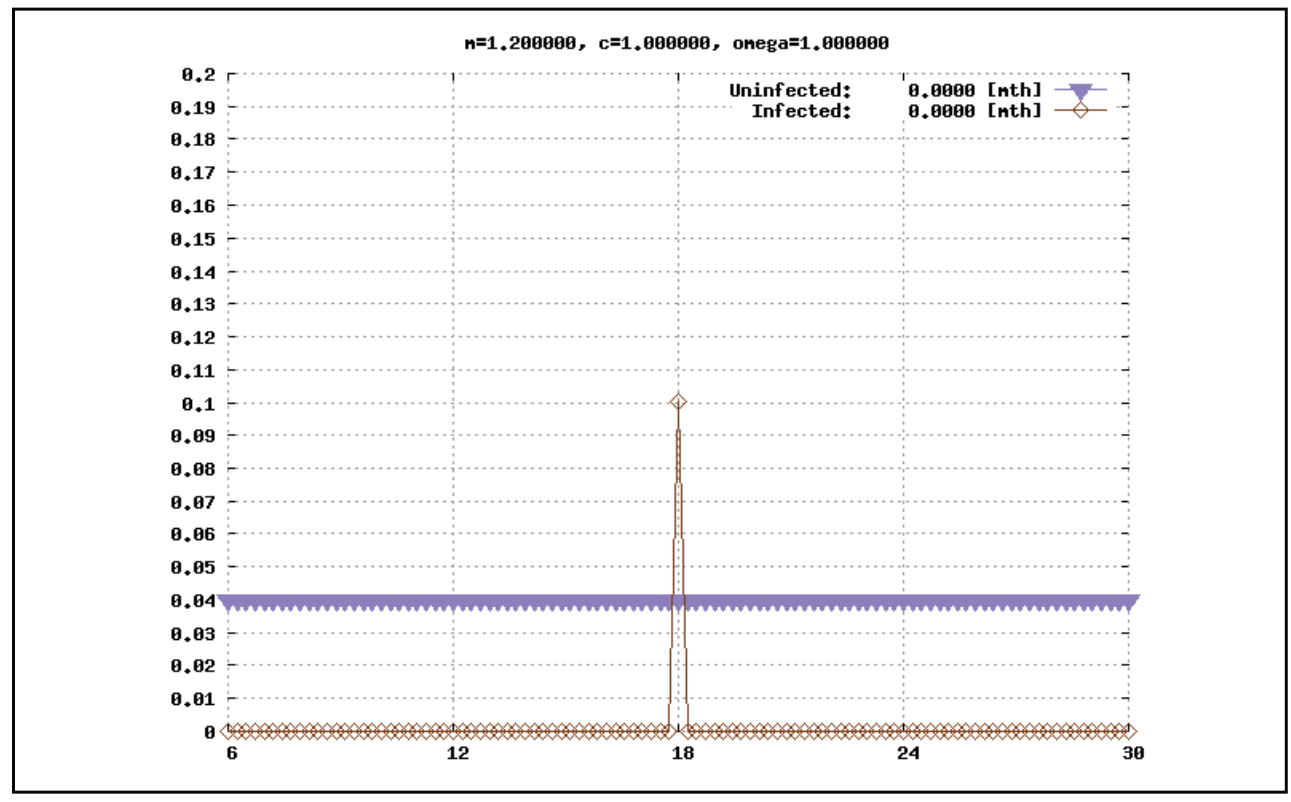

Figure 1: Profile of numerical solution for $q=6.0 \mathrm{~s}=30.0, c=1.0, \omega=1.0, m=1.2$, and $t=0.0[\mathrm{mth}$ ].

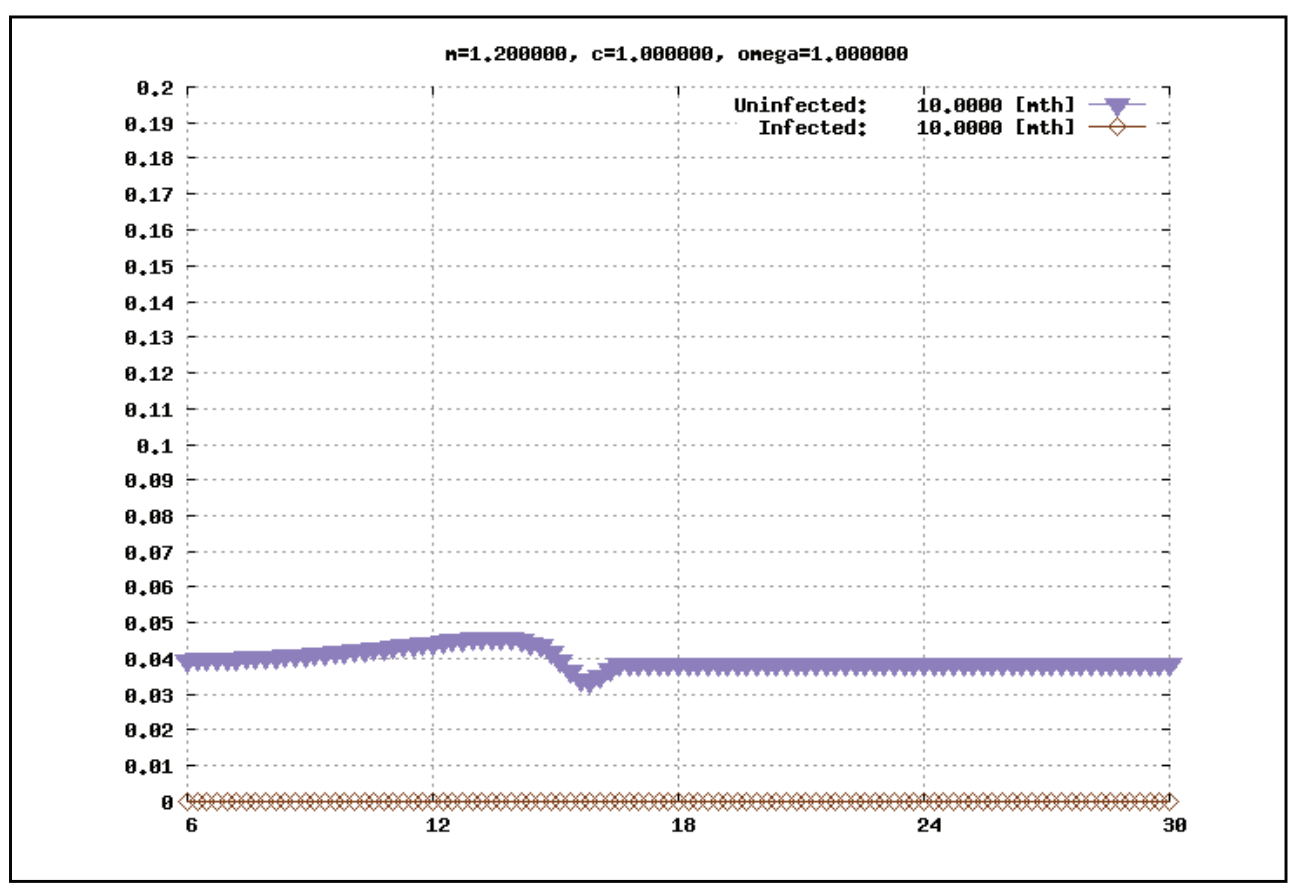

Figure 2: Profile of numerical solution for $q=6.0 \mathrm{~s}=30.0, c=1.0, \omega=1.0, m=1.2$, and $t=10$ [mth]. 


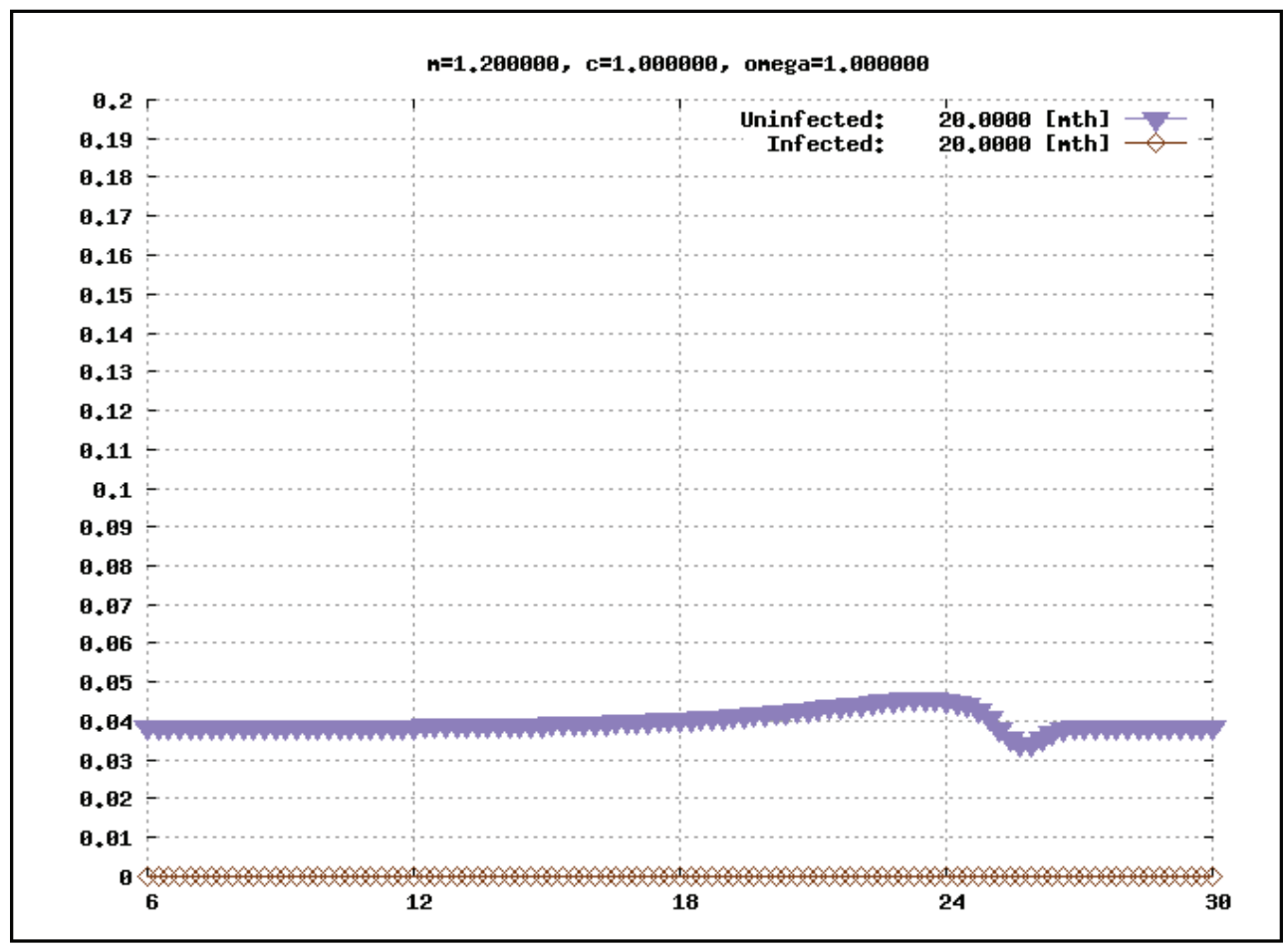

Figure 3: Profile of numerical solution for $q=6.0 \mathrm{~s}=30.0, c=1.0, \omega=1.0, m=1.2$, and $t=20[\mathrm{mth}$.

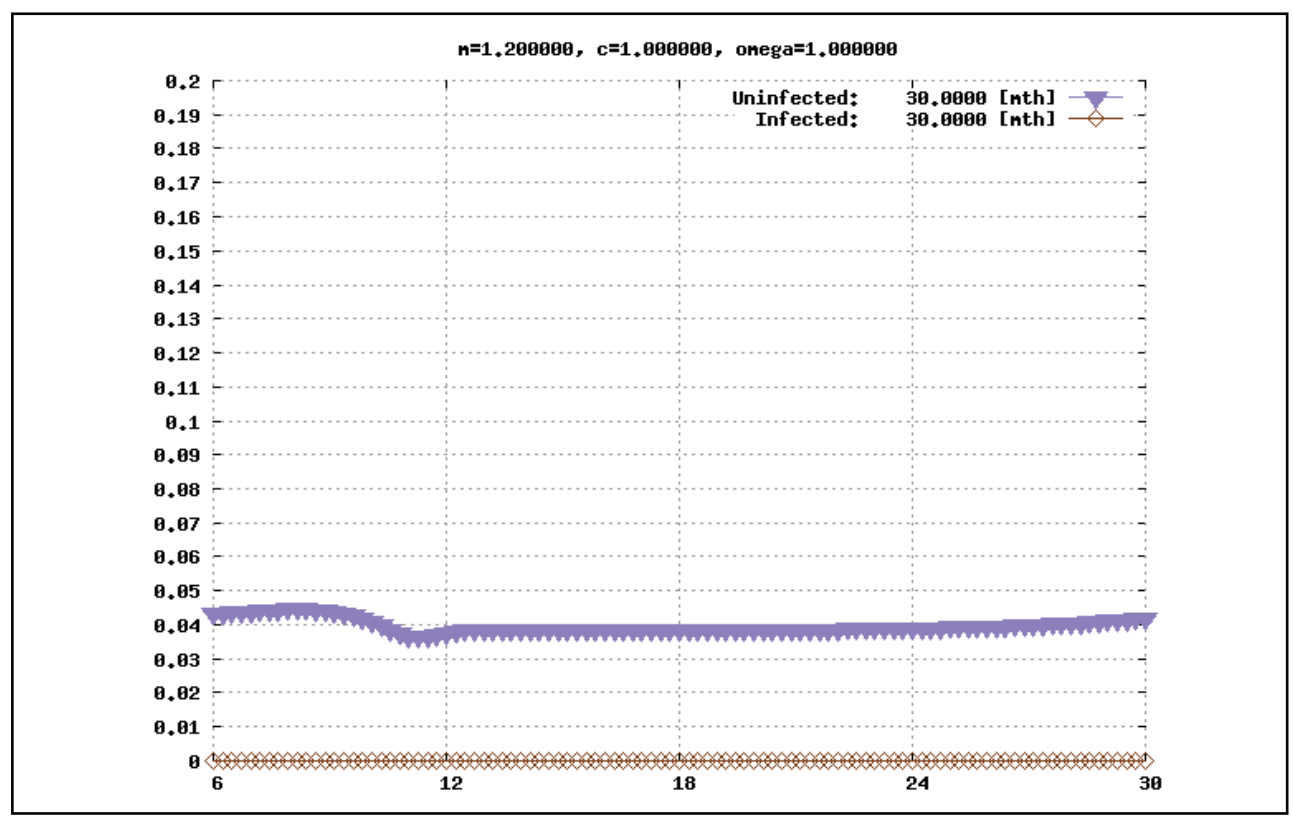

Figure 4: Profile of numerical solution for $q=6.0 \mathrm{~s}=30.0, c=1.0, \omega=1.0, m=1.2$, and $t=30[\mathrm{mth}]$. 


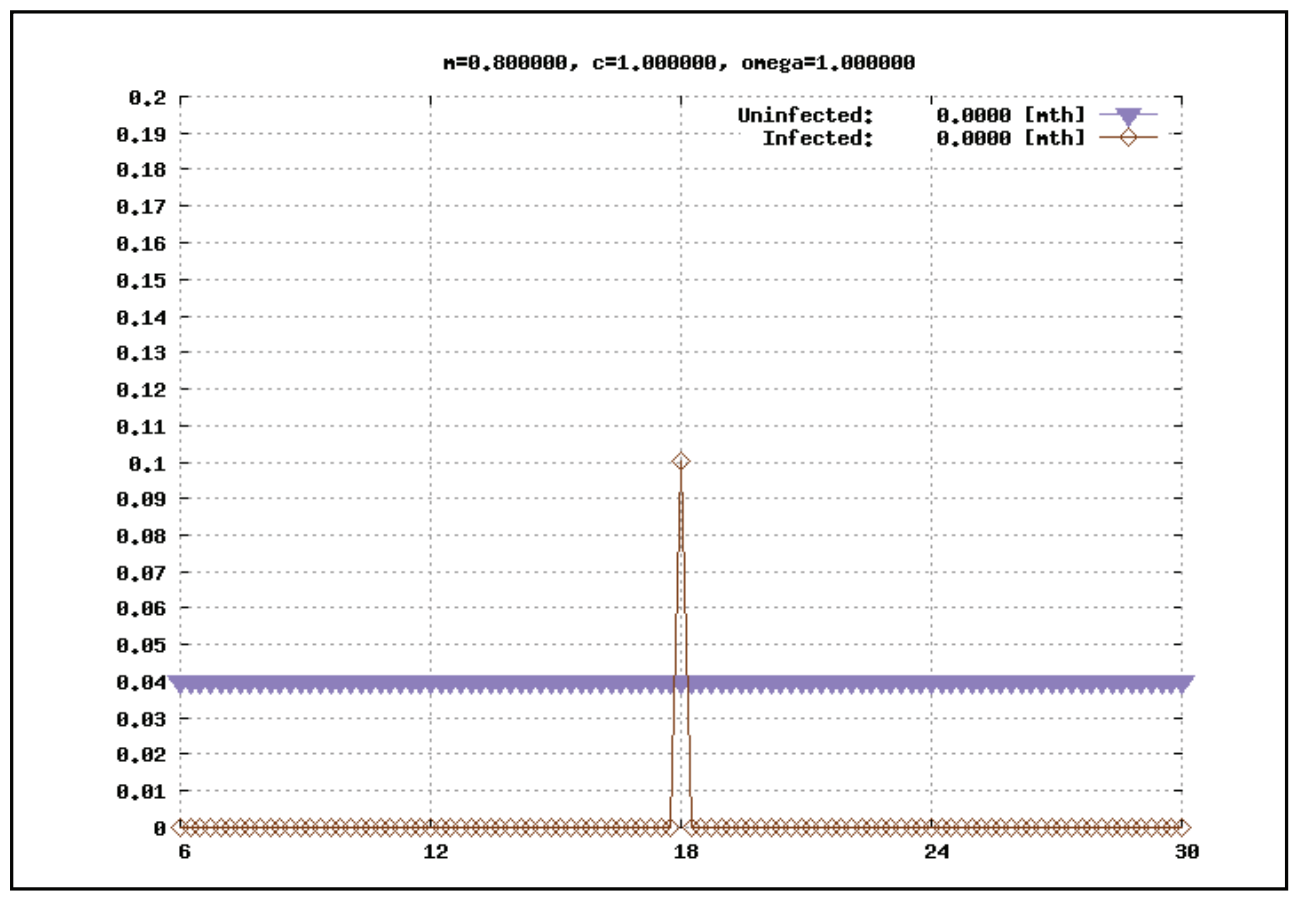

Figure 5: Profile of numerical solution for $q=6.0 \mathrm{~s}=30.0, c=1.0, \omega=1.0, m=0.8$, and $t=0.0[\mathrm{mth}]$

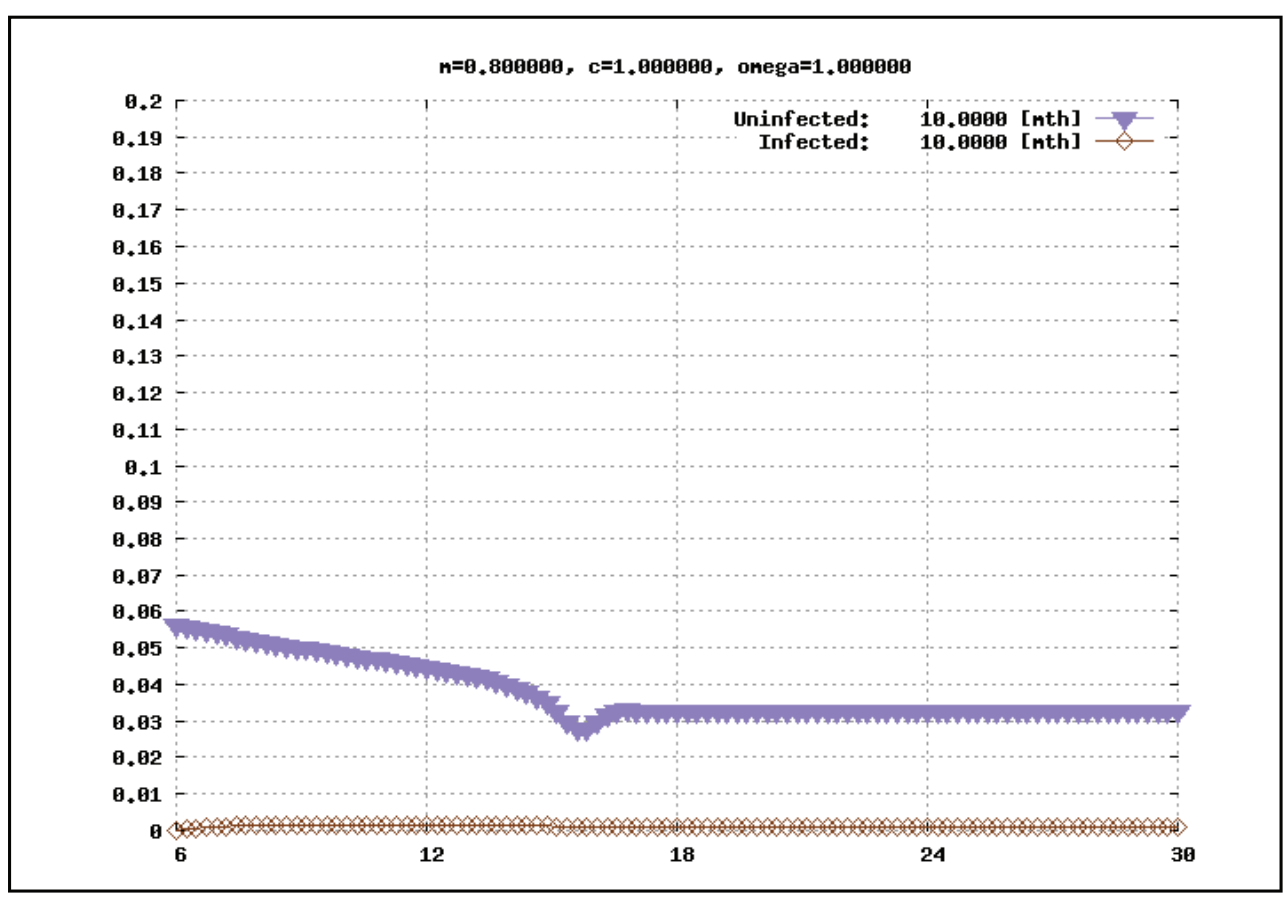

Figure 6: Profile of numerical solution for $q=6.0 \mathrm{~s}=30.0, c=1.0, \omega=1.0, m=0.8$, and $t=10[\mathrm{mth}]$. 


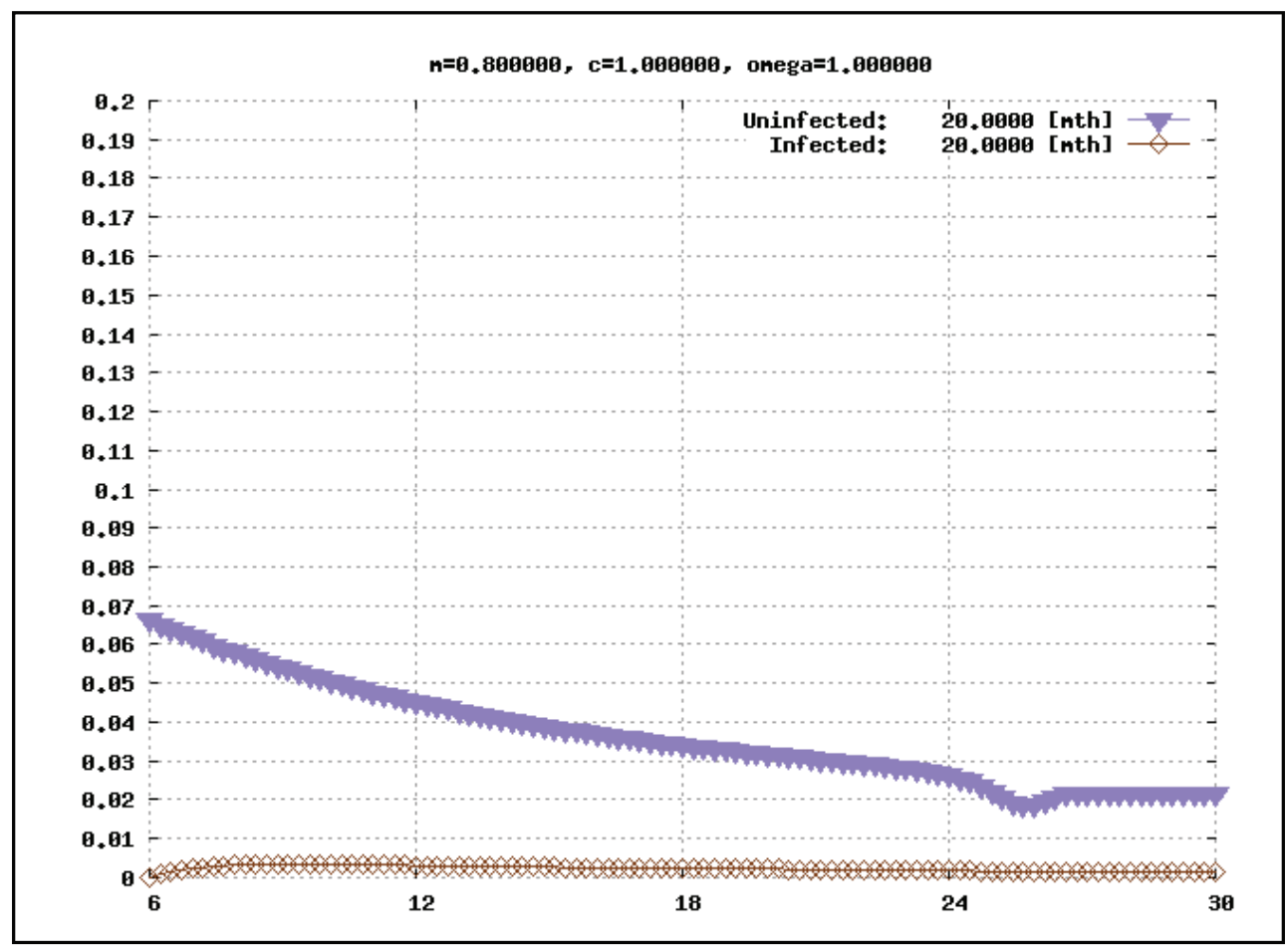

Figure 7: Profile of numerical solution for $q=6.0 \mathrm{~s}=30.0, c=1.0, \omega=1.0, m=0.8$, and $t=20[\mathrm{mth}$.

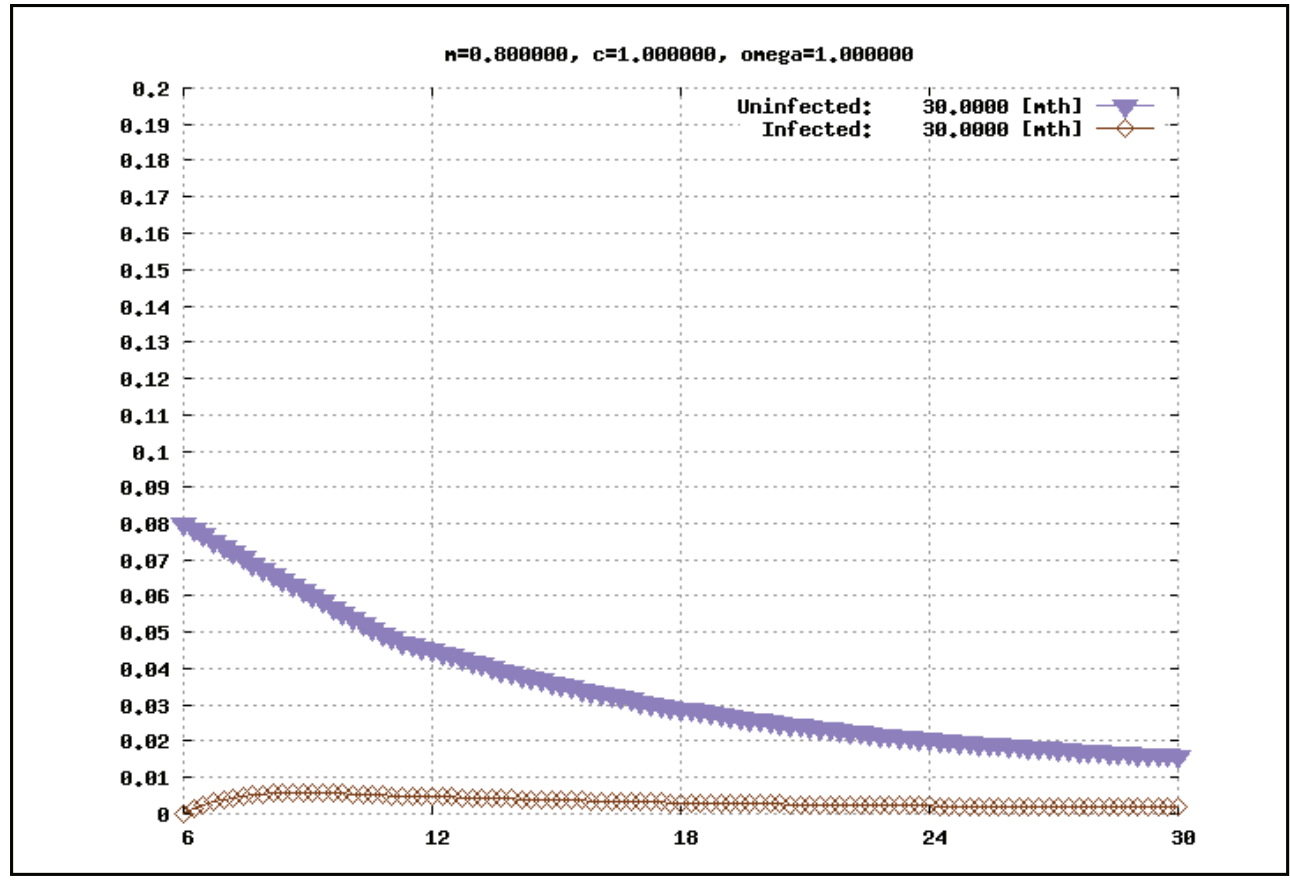

Figure 8: Profile of numerical solution for $q=6.0 \mathrm{~s}=30.0, c=1.0, \omega=1.0, m=0.8$, and $t=30$ [mth]. 


\section{Discussions}

The previous studies based on the mathematical model (1), (2) showed that the stationary solution corresponding to the infection free state was asymptotically stable while the other stationary solution was unpractical. In this study, age structure was incorporated into formulation of the mathematical model. Numerical solutions of the initial-boundary value problem (3) - (6) have shown that the constant solution corresponding to the infection free state is asymptotically stable for a relatively large value of the removal rate $m$. Numerical results indicated that the infection free state was unstable when another non-constant time independent solution was asymptotically stable.

Those results suggest that the condition $c \omega-m<0$ is a possible criterion for stability of the infection free state. Note that vaccination reduces the value of $\omega$. Our numerical results based on the age structured model have shown that removal of infected birds is essential, and that proper vaccination is also an important factor for security of a poultry farm against a bird flu outbreak.

\section{Acknowledgements}

The authors thank Dr. K. Yamamoto for his technical support. This work was supported by JSPS KAKENHI Grant Number 16 K05276.

\section{References}

[1] Earl A. Coddington and Norman Levinson, Theory of Ordinary Differential Equations, Robert E. Krieger Publishing Company, 1984, Malabar, Florida (Original Edition 1955, McGraw Hill Co., Inc.)

[2] Tertia Delia Nova, Herman Mawengkang, Masaji Watanabe, Modeling and Analysis of Bird Flu Outbreak within A Poultry Farm, Proceedings of the First International Conference on Bioinformatics, Editors: Ana Fred, Joaquim Filipe and Hugo Gamboa, 2010 INSTICC - Institute for Systems and Technologies of Information, Control and Communication (Short paper), 96-101, 2010. ISBN: 978-989-674-019-1

[3] T. Delia Nova, H. Mawengkang, and M. Watanabe, Study on control of bird flu outbreak within a poultry farm, In P. Howlett, M. Nelson, and A. J. Roberts, editors, Proceedings of the 9th Biennial Engineering Mathematics and Applications Conference, EMAC-2009, volume 51 of ANZIAM J., pages C668-C681, September 2010. http://journal.austms.org.au/ojs/index.php/ANZIAMJ/article/view/2432. ISSN 
$1446-8735$

[4] Masaji Watanabe, Tertia Delia Nova, Study on Bird Flu Infection Process within A Poultry Farm with Modeling and Simulation, Proceedings of the IASTED Asian Conference, Modelling, Identification, and Control (AsiaMIC 2012), April 2-4, Phuket, Thailand, Editor: W. Assawinchaichote. DOI: 10.2316/P.2012.769-059

[5] Tertia Delia Nova, Herman Mawengkang, Masaji Watanabe, Strategy Against Bird Flu Outbreak Within a Poultry Farm Based on Host-Virus Model, Proceedings of Dynamic Systems and Applications, Volume 6, EDITORS: G. S. Ladde, N. G. Medhin, Chuang Peng, M. Sambandham, Dynamic Publishers, Inc., U.S.A., (2012) 277-284. ISBN 1-890888-06-0

[6] Tertia Delia Nova, Arrival Rince Putri, Masaji Watanabe, Analytical Study on Bird Flu Transmission Process within Poultry Farm, Conference Proceedings, July 2015, Hokkaido, Japan, International Conference on Engineering and Applied Science (ICEAS), ISBN 978-986-87417-1-3, ICEAS-3888, 212-221.

[7] Matt J. Keeling and Pejman Rohani, Modeling infectious diseases in humans and animals, Princeton University Press, 2008, Princeton, New Jersey. 